\title{
Preoperative patient expectations and pain improvement after adult spinal deformity surgery
}

\author{
*Micheal Raad, MD, Andrew B. Harris, BS, Varun Puvanesarajah, MD, Mostafa H. El Dafrawy, MD, \\ Floreana N. Kebaish, MD, Brian J. Neuman, MD, Richard L. Skolasky, ScD, David B. Cohen, MD, \\ and Khaled M. Kebaish, MD
}

Department of Orthopaedic Surgery, The Johns Hopkins University, Baltimore, Maryland

\begin{abstract}
OBJECTIVE Patients' expectations for pain relief are associated with patient-reported outcomes after treatment, although this has not been examined in patients with adult spinal deformity (ASD). The aim of this study was to identify associations between patients' preoperative expectations for pain relief after ASD surgery and patient-reported pain at the 2-year follow-up.
\end{abstract}

METHODS The authors analyzed surgically treated ASD patients at a single institution who completed a survey question about expectations for back pain relief. Five ordinal answer choices to "I expect my back pain to improve" were used to categorize patients as having low or high expectations. Back pain was measured using the 10-point numeric rating scale (NRS) and Scoliosis Research Society-22r (SRS-22r) patient survey. Preoperative and postoperative pain were compared using analysis of covariance.

RESULTS Of 140 ASD patients eligible for 2-year follow-up, 105 patients (77 women) had pre- and postoperative data on patient expectations, 85 of whom had high expectations. The mean patient age was $59 \pm 12$ years, and 46 patients (44\%) had undergone previous spine surgery. The high-expectations and low-expectations groups had similar baseline demographic and clinical characteristics ( $p>0.05$ ), except for lower SRS-22r mental health scores in those with low expectations. After controlling for baseline characteristics and mental health, the mean postoperative NRS score was significantly better (lower) in the high-expectations group $(3.5 \pm 3.5)$ than in the low-expectations group $(5.4 \pm 3.7)(p=$ 0.049 ). The mean postoperative SRS-22r pain score was significantly better (higher) in the high-expectations group ( 3.3 $\pm 1.1)$ than in the low-expectations group $(2.6 \pm 0.94)(p=0.019)$.

CONCLUSIONS Despite similar baseline characteristics, patients with high preoperative expectations for back pain relief reported less pain 2 years after ASD surgery than patients with low preoperative expectations.

https://thejns.org/doi/abs/10.3171/2020.3.SPINE191311

KEYWORDS adult spinal deformity; back pain; health-related quality of life; patient expectations; patient-reported outcomes; Scoliosis Research Society-22r patient survey

$\mathrm{P}$ AIN is the most common symptom reported by patients who seek surgical treatment for adult spinal deformity (ASD). ${ }^{1,2}$ Although surgery has the potential to relieve the pain these patients experience, ${ }^{3,4}$ pain is a complex, multifactorial symptom that is influenced by modifiable intrinsic patient factors, such as fear, catastrophizing, self-efficacy, attitudes and beliefs, stress, and expectations for treatment. ${ }^{5-9}$

As healthcare becomes increasingly patient-centered, there has been an effort to understand the psychosocial factors that influence back pain. ${ }^{10-13}$ The Institute of Medicine recently published recommendations for pain treat- ment in its report Relieving Pain in America. ${ }^{14}$ Among the recommendations are an emphasis on intrinsic patient differences and variable responses to treatment. Although pain treatment for ASD patients is often accomplished through a multidisciplinary approach, it is essential for the surgeons who treat these patients to understand the complexity of pain perception and self-reported outcomes.

One of the intrinsic patient differences that can influence pain is the patient's expectations for treatment before receiving it. As defined in healthcare and quality improvement literature, patient expectations encompass ideal expectations (the patient's preferred outcome), predicted ex-

ABBREVIATIONS ASD = adult spinal deformity; NRS = numeric rating scale; ODI = Oswestry Disability Index; SRS-22r = Scoliosis Research Society-22r .

SUBMITTED November 1, 2019. ACCEPTED March 16, 2020.

INCLUDE WHEN CITING Published online June 12, 2020; DOI: 10.3171/2020.3.SPINE191311.

${ }^{*}$ M.R. and A.B.H. contributed equally to this work and share first authorship. 
pectations (the patient's anticipated outcome), normative expectations (what the patient thinks should happen), and unformed expectations (expectations that the patient is unable to articulate). ${ }^{15}$ Studies suggest that higher expectations for pain relief before treatment are correlated with lower levels of pain after various surgical and nonsurgical interventions. ${ }^{16-18}$ This correlation has also been reported in patients undergoing lumbar spine surgery, but with mixed results. ${ }^{19-22}$

All ASD patients have some degree of preformed expectations about what their surgery will accomplish. Thus, investigation of this integral construct of patient care can provide valuable information to spine surgeons and other members of the treatment team, potentially allowing risk stratification of patients according to their likelihood of anticipating positive postoperative improvement. The goals of this study were to characterize patients' preoperative expectations for pain relief after surgery for ASD and to examine associated self-reported improvements in pain after surgery. We hypothesized that preoperative patient expectations for pain relief would be associated with pain levels at the 2-year follow-up.

\section{Methods}

The study received approval from The Johns Hopkins University institutional review board.

\section{Patient Sample}

A database of patients treated by a spine surgeon at a large academic institution was queried to identify those who underwent corrective surgery for ASD from 2006 to 2014. Inclusion criteria were age $>18$ years, preoperative diagnosis of ASD, and availability of patient-reported outcome measures recorded preoperatively and at the 2-year follow-up. Our standard postoperative pain management regimen for these patients consists of a hydromorphone patient-controlled analgesic pump during the inpatient stay. Before discharge from the hospital, patients are transitioned to oral pain medications, typically including acetaminophen, gabapentin, cyclobenzaprine, and oxycodone. Most patients are not taking opioid pain medications for more than 6 weeks postoperatively. If patients have uncontrolled pain at 3 months postoperatively, they are referred to a pain management specialist.

\section{Assessment of Expectations for Back Pain Relief}

To assess expectations for back pain relief, we asked patients to choose 1 of 5 ordinal answers with which they most agreed regarding the statement, "I expect my back pain to improve." According to these results, we divided patients into 2 groups to provide dichotomous categorization of patients in a way that would be simple to integrate into routine clinical practice. In addition, the data regarding expectations had a bimodal distribution, which indicated that dichotomous categories would better represent patient activation levels than tripartite categories. Patients who answered "not at all," "slightly," or "somewhat likely" were classified as having low expectations for pain relief. Patients who answered "very" or "extremely likely" to experience relief from back pain were classified as having high expectations for pain relief. This question is similar to questions used in previous studies of patient expectations for pain relief after spine surgery. ${ }^{19,23}$

\section{Patient-Reported Outcome Measurements}

Pain was assessed using a numeric rating scale (NRS) and the Scoliosis Research Society-22r (SRS-22r) patient survey. The SRS-22r consists of 22 questions and is intended to assess health-related quality of life across 5 domains: function, mental health, pain, satisfaction, and self-image. Each domain is scored from 1 to 5, with higher scores indicating better outcomes. The SRS-22r is used widely to assess outcomes in ASD patients and has been shown to be valid, reliable, and responsive in this population. ${ }^{24-27}$ The NRS asks patients to rate the intensity of their current pain on a scale of 0 (no pain) to 10 (most pain). The NRS and other visual analog scales have been well validated for assessment of pain in patients who have undergone spine surgery. ${ }^{28-30}$ The Oswestry Disability Index (ODI) was also used to measure patient-reported disability. The ODI is graded on a scale ranging from 0 to 50 points, with a higher score indicating more severe disability, and has been shown to be a consistently valid tool for assessing patient outcomes. ${ }^{31}$

\section{Statistical Analysis}

Continuous variables are reported as means \pm standard deviation. Student t-tests were used to compare pairwise changes in radiographic measurements and patient-reported outcomes. The 2 groups were compared with respect to baseline demographic characteristics, radiographic deformity, surgical factors, and patient-reported outcomes using 2-tailed Student t-tests. The primary outcome was the difference in postoperative pain levels between the low-expectations and high-expectations groups, measured by one-way analysis of covariance, controlling for baseline factors, including preoperative SRS-22r mental health score and preoperative scores for each outcome assessed. These covariates were determined a priori to account for known variation in pain preoperatively and the potential interaction between mental health and pain perception. Significance was set at $\alpha=0.05$. Statistical analysis was performed using IBM SPSS software (version 25, IBM Corp.).

\section{Results}

\section{Patient and Surgical Characteristics}

We identified 250 patients in our database who underwent ASD surgery. Of these patients, 140 were eligible for 2-year clinical follow-up (surgical date $\geq 2$ years before the date of analysis). A total of 105 eligible patients (75\%) who had completed the 2-year clinical follow-up were included in the final cohort. The study population consisted of 77 women $(73 \%)$ and 28 men $(27 \%)$, with a mean age of 59 \pm 12 years. Participants had a mean of $9.5 \pm 3.5$ vertebrae instrumented. Forty-six patients (44\%) had undergone previous spine surgery. The mean baseline radiographic measures of the overall study population included a sagittal vertical axis $(\mathrm{C} 7-\mathrm{S} 1)$ of $9.6 \pm 9.8 \mathrm{~cm}$, thoracic kyphosis (T12-T4) of $36^{\circ} \pm 20^{\circ}$, and a thoracolumbar Cobb angle (T12-L4) of $23^{\circ} \pm 19^{\circ}$. 
TABLE 1. Baseline demographic and clinical characteristics of all patients and expectations for pain relief after surgery

\begin{tabular}{|c|c|c|c|c|}
\hline Characteristic & All Patients $(n=105)$ & Low Expectations $(n=20)$ & High Expectations $(n=85)$ & $\mathrm{p}$ Value \\
\hline Age, yrs & $59 \pm 12$ & $57 \pm 11$ & $59 \pm 12$ & 0.23 \\
\hline Female sex, n (\%) & $77(73)$ & $13(65)$ & $64(79)$ & 0.35 \\
\hline No. of instrumented vertebrae & $9.5 \pm 3.5$ & $9.7 \pm 3.5$ & $9.4 \pm 3.6$ & 0.79 \\
\hline Previous spine surgery, n (\%) & $46(44)$ & $8(40)$ & $38(45)$ & 0.70 \\
\hline 3-column osteotomy, n (\%) & $39(37)$ & $9(45)$ & $29(34)$ & 0.36 \\
\hline \multicolumn{5}{|l|}{ Radiographic deformity } \\
\hline Sagittal vertical axis, cm & $9.6 \pm 9.8$ & $11 \pm 8.4$ & $9.5 \pm 10$ & 0.67 \\
\hline Thoracic kyphosis, $^{\circ}$ & $36 \pm 20$ & $39 \pm 21$ & $36 \pm 20$ & 0.57 \\
\hline Thoracolumbar Cobb angle, ${ }^{\circ}$ & $23 \pm 19$ & $17 \pm 17$ & $15 \pm 20$ & 0.09 \\
\hline \multicolumn{5}{|l|}{ Patient-reported outcome measure } \\
\hline NRS for back pain & $7.3 \pm 3.4$ & $8.1 \pm 2.3$ & $7.1 \pm 3.6$ & 0.23 \\
\hline ODI score & $47 \pm 17$ & $52 \pm 19$ & $46 \pm 16$ & 0.20 \\
\hline \multicolumn{5}{|l|}{ SRS-22r } \\
\hline Pain & $2.3 \pm 0.83$ & $2.3 \pm 0.86$ & $2.4 \pm 0.83$ & 0.23 \\
\hline Physical function & $2.6 \pm 0.72$ & $2.5 \pm 0.69$ & $2.6 \pm 0.73$ & 0.90 \\
\hline Self-image & $2.4 \pm 0.79$ & $2.3 \pm 0.82$ & $2.4 \pm 0.79$ & 0.51 \\
\hline Mental health & $3.2 \pm 0.78$ & $2.9 \pm 0.80$ & $3.3 \pm 0.75$ & 0.02 \\
\hline Satisfaction & $2.7 \pm 1.0$ & $2.5 \pm 0.99$ & $2.8 \pm 1.0$ & 0.39 \\
\hline
\end{tabular}

Values are presented as the mean \pm SD unless stated otherwise.

\section{Expectations for Back Pain Relief}

Of the 105 patients, $85(81 \%)$ had high preoperative expectations for back pain relief after surgery, and 20 (19\%) had low expectations. The 2 groups were similar with respect to age, sex distribution, number of instrumented vertebrae, history of spine surgery, and whether a 3 -column osteotomy was performed (all $\mathrm{p}>0.05$ ). The 2 groups also had similar radiographic deformity and levels of back pain measured by the NRS and SRS-22r (all $\mathrm{p}$ $>0.05)$. The only variable that differed significantly between groups was the SRS-22r mental health score, which was lower (worse) in patients with low expectations (2.9 vs $3.3, \mathrm{p}=0.02)($ Table 1$)$.

\section{Patient-Reported Outcomes}

At the 2-year follow-up, 77 (73\%) patients reported pain improvement as measured by the NRS. After controlling for baseline characteristics, the mean postoperative NRS score was significantly better (lower) in the high-expectations group $(3.5 \pm 3.5)$ than in the low-expectations group $(5.4 \pm 3.7)(\mathrm{p}=0.049)$. Similarly, the mean postoperative SRS-22r pain score was significantly better (higher) in the high-expectations group $(3.3 \pm 1.1)$ than in the low-expectations group $(2.6 \pm 0.94)(\mathrm{p}=0.019)$. The ODI and other domains of the SRS-22r were not significantly different between the low-expectations and high-expectations groups (Table 2).

\section{Discussion}

Patients with high preoperative expectations for back pain relief after surgical correction of ASD reported less pain at the 2-year follow-up compared with those with low expectations, as measured by the NRS and SRS-22r. In our study, these 2 groups of patients had similar baseline characteristics, differing only with respect to SRS-22r mental health score. Moreover, the differences in pain (1.9-point difference in NRS score and 0.65-point difference in SRS-22r pain score) were clinically relevant. The minimum clinically important differences have been reported to be 0.24-0.57 for the SRS-22r pain score ${ }^{32}$ and $1.0-2.2$ for the NRS score. ${ }^{28,33}$ Our results not only support a growing body of evidence indicating the importance of patient-centered care, but also add support to the intuitive idea that patients with a positive mental attitude fare better than those with a negative mental attitude.

Studies analyzing the preoperative expectations for spine surgery patients have assessed several outcome measures and focused largely on lumbar spine surgery patients. A prospective study of 155 patients by Yee et al..$^{22}$ suggested that patients with higher preoperative expectations reported better Short Form-36 scores after lumbar spine surgery. McGregor et al..$^{20}$ conducted a similar study of 316 patients as part of a larger randomized controlled trial and found a moderate correlation between patient expectations and improvements in back and leg pain at 6 weeks, 6 months, and 12 months after lumbar spine surgery. In a recent study by Mancuso et al., ${ }^{19} 422$ patients who underwent lumbar spine surgery were asked preoperatively about their expectations for pain relief after surgery. The authors reported an inverse relationship between patient expectations for pain relief and patient-reported improvements in pain at the 2-year follow-up (i.e., patients who expected greater pain relief reported less improvement). The relationship 
TABLE 2. Changes in patient-reported outcomes from preoperatively to 2 years postoperatively, by expectations for pain relief

\begin{tabular}{ccccccc}
\hline \multirow{2}{*}{$\begin{array}{c}\text { Patient-Reported } \\
\text { Outcome Measure }\end{array}$} & \multicolumn{2}{c}{ Low Expectations } & & \multicolumn{2}{c}{ High Expectations } & \\
\cline { 2 - 3 } \cline { 5 - 6 } & Preop & Postop & & Preop & Postop & p Value $\dagger$ \\
\hline NRS for back pain* & $8.1 \pm 2.3$ & $5.4 \pm 3.7$ & & $7.1 \pm 3.6$ & $3.5 \pm 3.5$ & 0.049 \\
\hline ODI & $52 \pm 19$ & $37 \pm 24$ & & $46 \pm 16$ & $25 \pm 20$ & 0.532 \\
\hline SRS-22r & & & & & \\
\hline Pain & $2.3 \pm 0.86$ & $2.6 \pm 0.94$ & & $2.4 \pm 0.83$ & $3.3 \pm 1.1$ & 0.019 \\
\hline Physical function & $2.5 \pm 0.69$ & $3.3 \pm 0.69$ & & $2.6 \pm 0.73$ & $3.4 \pm 0.64$ & 0.644 \\
\hline Self-image & $2.3 \pm 0.82$ & $3.0 \pm 0.91$ & & $2.4 \pm 0.79$ & $3.1 \pm 1.1$ & 0.441 \\
\hline Mental health $\ddagger$ & $2.9 \pm 0.80$ & $3.2 \pm 0.67$ & & $3.3 \pm 0.75$ & $3.6 \pm 0.71$ & 0.064 \\
\hline Satisfaction & $2.5 \pm 0.99$ & $2.9 \pm 1.4$ & & $2.8 \pm 1.0$ & $3.3 \pm 1.5$ & 0.478 \\
\hline
\end{tabular}

* Scale ranges from 0 (no pain) to 10 points.

† Comparison of postoperative scores using analysis of covariance, controlling for baseline score variation and preoperative SRS-22r mental health score, as described in Methods.

$\ddagger$ Preoperative SRS-22r mental health score was excluded from covariates because of colinearity.

between preoperative expectations and pain relief reported in this study is unique among others in the literature and appears to be in contrast to our findings in ASD patients. However, the instrument used by Mancuso et al..$^{19}$ to assess expectations was also different from ours. The differences in instruments used to assess expectations for pain relief highlight the need for a validated tool to assess this patient-centered construct. It is important to note that most of the patients included in these studies were undergoing 1or 2-level arthrodesis for spinal stenosis, degenerative disc disease, or degenerative spondylolisthesis. ${ }^{19,20,22}$ Applying these results to ASD patients is complicated by the differences in pain generation between patients with ASD and degenerative lumbar spinal disorders and is complicated even further by evidence that suggests differences between expectations and patient-reported outcomes even between subgroups of patients who have undergone lumbar surgery. For example, Toyone et al. ${ }^{21}$ found a positive association between expectations and satisfaction in patients who underwent discectomy for lumbar disc herniation but no such association in patients who underwent laminotomy for spinal stenosis. Thus, these studies show that the interplay between expectations, condition being treated, and recovery is complex, and these results may or may not be generalizable to ASD patients.

We also found that patients in both the high-expectations and low-expectations groups had similar characteristics, except for lower SRS-22r mental health scores in the patients with low expectations for pain relief after surgery. Patients with depression and other mood disorders are known to have more pain and worse outcomes after spinal deformity surgery. ${ }^{34}$ Therefore, it is not surprising that the patients with low expectations and subsequently worse outcomes also scored lower on a mental health rating scale. The study by Mancuso et al. ${ }^{19}$ also found a correlation between screening positive for depression preoperatively and experiencing less improvement in pain after lumbar spinal surgery.

We believe that a patient's investment in their own care is important, particularly for ASD patients, whose postop- erative recovery process often requires substantial effort on the part of the patient. Future studies should explore the reasons behind patients' various expectations for surgery and whether interventions to change these expectations can affect outcomes. A recent cross-sectional study by Mancuso et al. ${ }^{35}$ attempted to identify how patients derive their expectations for lumbar surgery and found that the most common influences on patient expectations were their current surgeon (83\%), health-related information they read on the internet (55\%), social networks (26\%), other physicians (22\%), and previous health-related experiences $(65 \%)$. In addition, evidence indicates that interventions designed to raise preoperative patient expectations can affect surgical outcomes. ${ }^{17}$ Although factors that influence the expectations in ASD patients have yet to be defined, it seems that surgeons may have the greatest ability to influence patients' expectations for their surgical outcomes. Thus, we suggest that patients be risk stratified before surgery according to the likelihood of postoperative pain relief by using simple questions regarding selfreported expectations for pain relief. Surgeons and other healthcare providers may attempt to modify these expectations through in-depth discussion of the factors driving the expectations, as well as motivational interviewing techniques. However, prospective studies are still needed to examine the causal relationship between patients' preoperative expectations for pain relief and outcomes after ASD surgery, and whether interventions designed to improve these expectations can improve self-reported postoperative pain.

ASD can cause substantial disability, and the choice of treatment is often multifactorial. Patients often report poor perception of physical appearance, decreased physical function, poor mental health, disability, and pain. Although these issues can be improved by surgical treatment, the full dimensions of each domain must be appreciated to provide optimal care for patients with ASD. Improvements in patient-reported outcomes are often subjective and vary widely among patients. Thus, it is important that patients with ASD have realistic expectations for postop- 
erative relief of symptoms because their expectations may affect how they eventually view the success of their treatment. The importance of adequate preoperative counseling cannot be overstated, and more research is needed to define how various methods of informing patients of the risks and benefits of treatment may affect their expectations and subsequent perceptions of pain improvement.

This study has several limitations. First, as a retrospective study, it is inherently limited by information and selection bias, given that we only studied patients who had 2 years of follow-up. Second, although several similar methods of assessing patients' expectations for spine surgery have been used, ${ }^{23}$ instruments to measure patients' expectations for pain relief in this population have not been rigorously validated. Third, we did not fully characterize the patients' preoperative and postoperative pain, which may have changed in more ways than severity. Fourth, this study was conducted at a tertiary spine center and may not be generalizable to patients treated in different settings. Fifth, we were unable to assess opioid consumption, which may modulate patients' perceptions of postoperative pain. Lastly, a power analysis was not performed to determine sufficient sample size to find differences among all outcomes assessed. Therefore, some significant associations may not have been detected by our study. Despite these limitations, our study has the strengths of 2 years' follow-up in a large group of patients with ASD. To our knowledge, studies examining the relationship between the preoperative expectations and postoperative pain relief in ASD patients have not been reported.

\section{Conclusions}

Before undergoing surgery for ASD, patients have varied expectations for how their symptoms will change after surgery. Our study indicates that ASD patients who have high preoperative expectations for pain relief after surgery report lower pain levels at the 2-year follow-up than those who have low expectations. This finding supports the idea that pain in ASD patients is complex and mediated in part by psychosocial factors that should be addressed and discussed through preoperative patient education.

\section{References}

1. Glassman SD, Schwab FJ, Bridwell KH, et al. The selection of operative versus nonoperative treatment in patients with adult scoliosis. Spine (Phila Pa 1976). 2007;32(1):93-97.

2. Smith JS, Fu KM, Urban P, Shaffrey CI. Neurological symptoms and deficits in adults with scoliosis who present to a surgical clinic: incidence and association with the choice of operative versus nonoperative management. J Neurosurg Spine. 2008;9(4):326-331.

3. Scheer JK, Smith JS, Clark AJ, et al. Comprehensive study of back and leg pain improvements after adult spinal deformity surgery: analysis of 421 patients with 2-year follow-up and of the impact of the surgery on treatment satisfaction. J Neurosurg Spine. 2015;22(5):540-553.

4. Verma R, Lafage R, Scheer J, et al. Improvement in back and leg pain and disability following adult spinal deformity surgery: study of 324 patients with 2-year follow-up and the impact of surgery on patient-reported outcomes. Spine (Phila Pa 1976). 2019;44(4):263-269.

5. Bishop MD, Bialosky JE, Cleland JA. Patient expectations of benefit from common interventions for low back pain and effects on outcome: secondary analysis of a clinical trial of manual therapy interventions. J Manual Manip Ther. 2011;19(1):20-25.

6. Hirsh AT, George SZ, Bialosky JE, Robinson ME. Fear of pain, pain catastrophizing, and acute pain perception: relative prediction and timing of assessment. J Pain. 2008;9(9):806812.

7. Linton SJ. Do psychological factors increase the risk for back pain in the general population in both a cross-sectional and prospective analysis? Eur J Pain. 2005;9(4):355-361.

8. Turner JA, Ersek M, Kemp C. Self-efficacy for managing pain is associated with disability, depression, and pain coping among retirement community residents with chronic pain. J Pain. 2005;6(7):471-479.

9. Waddell G. Biopsychosocial analysis of low back pain. Baillieres Clin Rheumatol. 1992;6(3):523-557.

10. Morton L, de Bruin M, Krajewska M, et al. Beliefs about back pain and pain management behaviours, and their associations in the general population: a systematic review. Eur J Pain. 2019;23(1):15-30.

11. Nishigami T, Wand BM, Newport R, et al. Embodying the illusion of a strong, fit back in people with chronic low back pain. A pilot proof-of-concept study. Musculoskelet Sci Pract. 2019;39:178-183.

12. O'Keeffe M, George SZ, O'Sullivan PB, O'Sullivan K. Psychosocial factors in low back pain: letting go of our misconceptions can help management. Br J Sports Med. 2019;53(13):793-794.

13. Ramond-Roquin A, Bouton C, Gobin-Tempereau AS, et al. Interventions focusing on psychosocial risk factors for patients with non-chronic low back pain in primary care-a systematic review. Fam Pract. 2014;31(4):379-388.

14. Institute of Medicine. Relieving Pain in America: A Blueprint for Transforming Prevention, Care, Education, and Research. The National Academies Press; 2018.

15. Thompson AGH, Suñol R. Expectations as determinants of patient satisfaction: concepts, theory and evidence. Int $J$ Qual Health Care. 1995;7(2):127-141.

16. Linde K, Witt CM, Streng A, et al. The impact of patient expectations on outcomes in four randomized controlled trials of acupuncture in patients with chronic pain. Pain. 2007;128(3):264-271.

17. Rief W, Shedden-Mora MC, Laferton JA, et al. Preoperative optimization of patient expectations improves long-term outcome in heart surgery patients: results of the randomized controlled PSY-HEART trial. BMC Med. 2017;15(1):4.

18. Sherman KJ, Cherkin DC, Ichikawa L, et al. Treatment expectations and preferences as predictors of outcome of acupuncture for chronic back pain. Spine (Phila Pa 1976). 2010;35(15):1471-1477.

19. Mancuso CA, Reid MC, Duculan R, Girardi FP. Improvement in pain after lumbar spine surgery: the role of preoperative expectations of pain relief. Clin J Pain. 2017;33(2):9398.

20. McGregor AH, Doré CJ, Morris TP. An exploration of patients' expectation of and satisfaction with surgical outcome. Eur Spine J. 2013;22(12):2836-2844.

21. Toyone T, Tanaka T, Kato D, et al. Patients' expectations and satisfaction in lumbar spine surgery. Spine (Phila Pa 1976). 2005;30(23):2689-2694.

22. Yee A, Adjei N, Do J, et al. Do patient expectations of spinal surgery relate to functional outcome? Clin Orthop Relat Res. 2008;466(5):1154-1161.

23. Mancuso CA, Duculan R, Cammisa FP, et al. Fulfillment of patients' expectations of lumbar and cervical spine surgery. Spine J. 2016;16(10):1167-1174.

24. Baldus C, Bridwell KH, Harrast J, et al. Age-gender matched comparison of SRS instrument scores between adult defor- 
mity and normal adults: are all SRS domains disease specific? Spine (Phila Pa 1976). 2008;33(20):2214-2218.

25. Berven S, Deviren V, Demir-Deviren S, et al. Studies in the modified Scoliosis Research Society Outcomes Instrument in adults: validation, reliability, and discriminatory capacity. Spine (Phila Pa 1976). 2003;28(18):2164-2169.

26. Bridwell KH, Berven S, Glassman S, et al. Is the SRS-22 instrument responsive to change in adult scoliosis patients having primary spinal deformity surgery? Spine (Phila $\mathrm{Pa}$ 1976) 2007;32(20):2220-2225.

27. Bridwell KH, Cats-Baril W, Harrast J, et al. The validity of the SRS-22 instrument in an adult spinal deformity population compared with the Oswestry and SF-12: a study of response distribution, concurrent validity, internal consistency, and reliability. Spine (Phila Pa 1976). 2005;30(4):455-461.

28. Childs JD, Piva SR, Fritz JM. Responsiveness of the numeric pain rating scale in patients with low back pain. Spine (Phila Pa 1976). 2005;30(11):1331-1334.

29. Young IA, Cleland JA, Michener LA, Brown C. Reliability, construct validity, and responsiveness of the neck disability index, patient-specific functional scale, and numeric pain rating scale in patients with cervical radiculopathy. Am J Phys Med Rehabil. 2010;89(10):831-839.

30. Zanoli G, Strömqvist B, Jönsson B. Visual analog scales for interpretation of back and leg pain intensity in patients operated for degenerative lumbar spine disorders. Spine (Phila Pa 1976). 2001;26(21):2375-2380.

31. Fairbank JCT, Pynsent PB. The Oswestry Disability Index. Spine (Phila Pa 1976). 2000;25(22):2940-2952.

32. Crawford CH III, Glassman SD, Bridwell KH, et al. The minimum clinically important difference in SRS-22R total score, appearance, activity and pain domains after surgical treatment of adult spinal deformity. Spine (Phila Pa 1976). 2015;40(6):377-381.
33. Salaffi F, Stancati A, Silvestri CA, et al. Minimal clinically important changes in chronic musculoskeletal pain intensity measured on a numerical rating scale. Eur J Pain. 2004;8(4):283-291.

34. Theologis AA, Ailon T, Scheer JK, et al. Impact of preoperative depression on 2-year clinical outcomes following adult spinal deformity surgery: the importance of risk stratification based on type of psychological distress. J Neurosurg Spine. 2016;25(4):477-485.

35. Mancuso CA, Duculan R, Cammisa FP, et al. Sources of patients' expectations of lumbar surgery. Spine (Phila Pa 1976). 2019;44(5):318-324.

\section{Disclosures}

Dr. Neuman: support of non-study-related clinical or research effort from DePuy Synthes and educational speaker for Medtronic.

\section{Author Contributions}

Conception and design: KM Kebaish, Raad, Skolasky. Analysis and interpretation of data: Raad, Harris. Drafting the article: Raad, Harris, Puvanesarajah, El Dafrawy, FN Kebaish, Neuman, Cohen. Critically revising the article: Raad, Harris, Puvanesarajah, El Dafrawy, FN Kebaish, Neuman, Cohen. Reviewed submitted version of manuscript: all authors. Approved the final version of the manuscript on behalf of all authors: KM Kebaish. Study supervision: KM Kebaish.

\section{Correspondence}

Khaled M. Kebaish: The Johns Hopkins University, Baltimore, MD. editorialservices@jhmi.edu. 